Cite this: Phys. Chem. Chem. Phys., 2011, 13, 17084-17092

\title{
Photoinduced hole trapping in single semiconductor quantum dots at specific sites at silicon oxide interfaces
}

\author{
Cornelius Krasselt,* Jörg Schuster† and Christian von Borczyskowski
}

Received 22nd June 2011, Accepted 28th July 2011

DOI: $10.1039 / \mathrm{c1cp22040c}$

Blinking dynamics of $\mathrm{CdSe} / \mathrm{ZnS}$ semiconductor quantum dots (QD) are characterized by (truncated) power law distributions exhibiting a wide dynamic range in probability densities and time scales both for off- and on-times. QDs were immobilized on silicon oxide surfaces with varying grades of hydroxylation and silanol group densities, respectively. While the off-time distributions remain unaffected by changing the surface properties of the silicon oxide, a deviation from the power law dependence is observed in the case of on-times. This deviation can be described by a superimposed single exponential function and depends critically on the local silanol group density. Furthermore, QDs in close proximity to silanol groups exhibit both high average photoluminescence intensities and large on-time fractions. The effect is attributed to an interaction between the QDs and the silanol groups which creates new or deepens already existing hole trap states within the $\mathrm{ZnS}$ shell. This interpretation is consistent with the trapping model introduced by Verberk et al. (R. Verberk, A. M. van Oijen and M. Orrit, Phys. Rev. B, 2002, 66, 233202).

\section{Introduction}

Single quantum objects show intriguing electronic and optical properties which became observable since the successful spectroscopy on such single systems. ${ }^{1,2}$ One fundamental and characteristic feature is the luminescence intermittency often denoted as blinking which was verified for single emitters like quantum dots (QDs), ${ }^{3-5}$ dye molecules, ${ }^{6-8}$ fluorescent proteins ${ }^{9}$ or even nitrogen-vacancy centres in nanodiamonds. ${ }^{10}$ Moreover, blinking is also observed in large organic systems like individual chains of conjugated polymers ${ }^{11,12}$ or J-aggregates. ${ }^{13}$ The phenomenon relates to fluctuating emission intensities between dark "off" and bright "on" events but is also characterized by intermediate states with intensities in-between. ${ }^{14,15}$ In the case of single quantum dots (and also dyes) the distributions for both on- and off-times follow most often power laws showing a huge dynamic range in times from several tens of nanoseconds up to minutes and in probability densities with even more orders of magnitude. ${ }^{16,17}$ Thus, blinking dynamics depend both on the QDs itself but also on their local environment. Namely, they are influenced by the particles' structure (material, shape, multiple layers), ${ }^{18-20}$ its passivation ${ }^{21}$ and occupation by ligands $\mathrm{s}^{20,22}$ on the one hand and by external conditions

Institute of Physics and nanoMA (Centre for nanostructured Materials and Analytics), Chemnitz University of Technology, 09107 Chemnitz, Germany.

E-mail: cornelius.krasselt@physik.tu-chemnitz.de;

Fax: + 49 (0)371531 837174; Tel: + 49 (0)371 53137174

$\dagger$ Now at: Fraunhofer Institute for Electronic Nano Systems (ENAS), Technologie-Campus 3, 09126 Chemnitz, Germany. like the surrounding matrix and its polarity, ${ }^{23-25}$ the atmosphere ${ }^{26,27}$ or electrical fields ${ }^{28,29}$ on the other. To explain this behaviour, several models have been proposed ${ }^{16,25,30-34}$ but especially the trapping model ${ }^{35}$ accounts for most features of fluorescence intermittency and the related huge dynamics. According to this model it is generally assumed, that photoinduced charging originates from tunnelling of the electron and/or hole of an exciton to trap states or self-induced trap states even for very long times. The photoluminescence of a subsequently excited new exciton will be (partly) quenched due to Auger relaxation or other non-radiative decay channels such as increased electron-phonon coupling. ${ }^{16}$ If the trap states are widely distributed owing to variations of tunnelling barriers and/or tunnelling distances, dynamics are readily described by (truncated) power laws. In this manner the power law dynamics of off-times can be explained in accordance with the existence of multiple trapping sites. Moreover, energetic or spatial disorder seems to be a prerequisite of (power law type) blinking. ${ }^{16}$

However, the discussion related to blinking is not settled and open questions still remain. In particular, the mechanisms responsible for the dynamics of the on-events have not been decoded conclusively so far. In this case, again, most experiments indicate a power law though the rate of deactivation of an initial state should be proportional to the sum of all parallel decay routes resulting in only one unique on-time (exponential dynamics). The clearly observed deviation from this assumption implies that either the trap state distribution is (slowly) fluctuating in time or that a second (independent) switching process has to be invoked. Verberk et al. already suggested ${ }^{35}$ that both, 
electron and hole, might be subject to tunnelling and trapping processes. This model has been extended recently by Chon et al., ${ }^{20}$ who show evidence that on-times depend on shell and ligands which influence hole and electron tunnelling. However, definite elaboration of power law dynamics needs a broad dynamic range over several orders of magnitude both in time and probability. In many reported experiments this condition is not fulfilled, which subsequently lacks definite assignment of the underlying models.

In the present paper we aim at precise determination of the off- and especially the on-times over a large dynamic range. We emphasize the complexity of the on-time dynamics by presenting measurements on the luminescence intermittency of $\mathrm{CdSe} / \mathrm{ZnS}$ QDs, initially capped with trioctylphosphine oxide (TOPO) ligands, on thermally grown silicon oxide of tuneable surface properties. It turns out that the often observed (truncated) on-time power law is superimposed by an additional single exponential function which correlates with the grade of hydroxylation of the silicon oxide surface as well as with the photoluminescence (PL) intensity. Furthermore, this behaviour varies for different single QDs. The hydroxylation is controlled by the density of silanol groups $(\mathrm{Si}-\mathrm{OH})$ which occur if hydroxyl groups, available from abundant water, bind to silicon atoms on the silicon oxide surface according to

$$
\mathrm{Si}-\mathrm{O}-\mathrm{Si}+\mathrm{H}_{2} \mathrm{O} \leftrightharpoons 2 \mathrm{Si}-\mathrm{OH} \text {. }
$$

These groups appear in different types like isolated, paired (geminal) or vicinal silanols and form islands of various sizes. $^{36}$ They may play an important role for the optical properties of QDs as they can also be negatively charged by deprotonation $\left(\mathrm{SiO}^{-}\right)$in the presence of water which leads to the formation of hydronium groups $\left(\mathrm{H}_{3} \mathrm{O}^{+}\right)^{37}$ as

$$
\mathrm{Si}-\mathrm{OH}+\mathrm{H}_{2} \mathrm{O} \leftrightharpoons \mathrm{SiO}^{-}+\mathrm{H}_{3} \mathrm{O}^{+} \text {. }
$$

Thus, (deprotonated) silanols influence their local environment by electrostatic interactions or as possible (static) trap states for charge carriers, possibly affecting the blinking dynamics of nearby QDs. The density of the silanol groups can be controlled by the preparation conditions of the silicon oxide. Annealing decreases the density of these groups whereby the vicinal silanols desorb at temperatures of $180-400{ }^{\circ} \mathrm{C}$, followed by the paired and isolated ones which vanish completely at temperatures of about 850 and $1200{ }^{\circ} \mathrm{C}$, respectively. ${ }^{36}$ The complete rehydroxylation of silicon oxide, i.e. the recovery of the original silanol density, is enforced through treatment with hydrolyzing compounds like Piranha or boiling water. ${ }^{36,38}$ In this way average densities varying by a factor of about 20 , namely from $\approx 0.25 \mathrm{OH} \mathrm{nm}^{-2}\left(T \approx 1000{ }^{\circ} \mathrm{C}\right)$ up to $\approx 4.6 \mathrm{OH} \mathrm{nm}{ }^{-2}$ (fully hydroxylated, $T \leq 180{ }^{\circ} \mathrm{C}$ ) were obtained. $^{36}$ The presented results for on-time contributions are discussed in the context of the above mentioned charge trapping model for single quantum objects and are attributed to hole trapping at surface sites at which silanol groups bind to the surface of the QDs.

\section{Experimental}

We used CdSe/ZnS core-shell QDs, capped with TOPO ligands, which were obtained in a $10^{-6} \mathrm{M}$ solution in toluene from Invitrogen. In ensemble measurements the particles emit at $565 \mathrm{~nm}$ with a FWHM of about $33 \mathrm{~nm}$. Single particle conditions for optical spectroscopy were fulfilled by further dilution of QDs in toluene and a subsequent immobilisation of the particles on cleaned silicon oxide surfaces by spincoating. The oxide, provided by the Centre for Microtechnologies, Chemnitz University of Technology, was thermally grown to a thickness of $100 \mathrm{~nm}$ on silicon wafers at temperatures about $1000{ }^{\circ} \mathrm{C}$ in oxygen atmosphere. Thus, the initial density of silanol groups on the oxide surfaces is low. By treatment with Piranha (a 4:6 mixture of $30 \%$ hydrogen peroxide and $96 \%$ sulfuric acid) in an ultrasonic bath at $80{ }^{\circ} \mathrm{C}$ for at least one hour the oxide became highly hydroxylated, leading to a high density of silanol groups of all three types (isolated, geminal, vicinal). To lower this density in a controlled way, a moderate and strong annealing of the silicon oxide at temperatures of about $500{ }^{\circ} \mathrm{C}(30 \mathrm{~min})$ and $800{ }^{\circ} \mathrm{C}(22 \mathrm{~h})$ or $1000{ }^{\circ} \mathrm{C}(10 \mathrm{~min})$ was performed at ambient conditions prior to spincoating of QDs. Whereas only the vicinal silanol groups vanish in the first case, a few isolated silanols remain on the surface in the latter annealing procedures. ${ }^{36}$ A subsequent Piranha treatment enforces the rehydroxylation of the silicon oxide surface and recovers the high silanol group density.

The luminescence time traces were recorded with a home-built wide field microscope consisting essentially of a high numerical aperture objective (100x/0.9NA, Zeiss) and a back-illuminated EMCCD camera (iXon, Andor). For excitation the $476 \mathrm{~nm}$ cw-line of an argon/krypton ion laser was used at average intensities of about $25 \mathrm{~W} \mathrm{~cm}^{-2}$.

\section{Results and discussion}

To analyze the blinking behaviour of $\mathrm{CdSe} / \mathrm{ZnS}$ core-shell QDs we recorded time traces of the emission of single nanocrystals for $30 \mathrm{~min}$ with an exposure time of $50 \mathrm{~ms}$. To cover a wide range of blinking dynamics (of about four and six orders of magnitude in time and probability density, respectively) we have investigated at least 100 single QDs on each examined silicon oxide surface with a given density of silanol groups. This high number of QDs ensures that the influence of a given environment on the blinking behaviour can be evaluated with extremely high sensitivity. The time traces were analyzed individually by separating the on- and off-events through a well determined and low lying intensity threshold defining the background noise as off-state without losing low-intensity on-states. On/off-time distributions, exhibiting the (scaled) probability density $p_{i}\left(t_{i}\right)$ of an event as a function of its length $t_{i}$, were obtained from histograms for the on/off-event durations where a linearly increasing bin size was used to flatten the statistics. Fig. 1 shows typical examples for both event distributions for QDs on Piranha-treated, highly hydroxylated silicon oxide, that is with a large silanol group density.

As known from literatures ${ }^{4,16,23}$ the off-time statistics follow a power law truncated by an exponential function ${ }^{31}$ (solid line in Fig. 1(a)) according to

$$
p_{\text {off }}\left(t_{\text {off }}\right) \propto t_{\text {off }}^{-\alpha_{\text {off }}} e^{-\Gamma_{\text {off }} \cdot t_{\text {off }}} .
$$

The power law exponent $\alpha_{\text {off }}$ and the truncation rate $\Gamma_{\text {off }}$ are found to be $1.71 \pm 0.03$ and $(21 \pm 3) \times 10^{-3} \mathrm{~s}^{-1}$ and agree with 

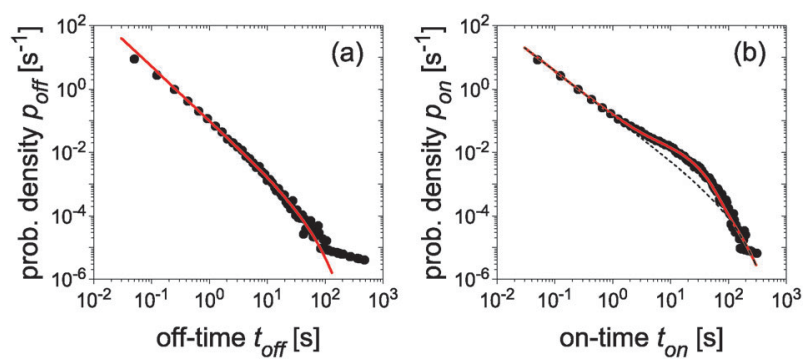

Fig. 1 Distributions for (a) off- and (b) on-times of $100 \mathrm{CdSe} / \mathrm{ZnS}$ QDs on Piranha-treated, highly hydroxylated silicon oxide. The data in (a) and (b) are fitted by a truncated power law according to eqn (3) and its extended version with an additional exponential function given in eqn (4), respectively. The dashed line in (b) denotes the best approximation according to eqn (3).

recently reported values for this system..$^{23,30,39}$ It should be mentioned that we have always scaled the probability density to the third data point corresponding to an event duration of $250 \mathrm{~ms}$. We have defined this point as the first stable data point due to the underestimation of the occurrence of shorter off-times. This results from the low lying intensity threshold which misses such (short) off-events if they do not match the integration time window of the measurement.

Regarding the on-time distribution one clearly discovers a deviation ("hump") from the recently published on-time histograms which can usually be described analogous to the off-times by the above given function of a truncated power law (dotted curve in Fig. 1(b)). 5,16,40,41 This on-time "hump" is described here for the first time although it is evident from several other publications related to blinking of $\mathrm{CdSe} / \mathrm{ZnS}$ QDs which do not dwell on this phenomenon. ${ }^{39,42,43}$ It emerges for times between 1 to $100 \mathrm{~s}$ with a maximum deviation up to a factor of 3 in probability density close to $20 \mathrm{~s}$ and can be characterized by an additional, superimposed exponential term complementing the truncated power law dependence (solid curve in Fig. 1(b)) and resulting in

$$
p_{\text {on }}\left(t_{\text {on }}\right)=A p_{A} t_{\text {on }}^{-\alpha_{\text {on }}} e^{-\Gamma_{\text {on }} \cdot t_{\text {on }}}+B p_{B} \mathrm{e}^{-\gamma_{\text {on }} \cdot t_{\text {on }}} .
$$

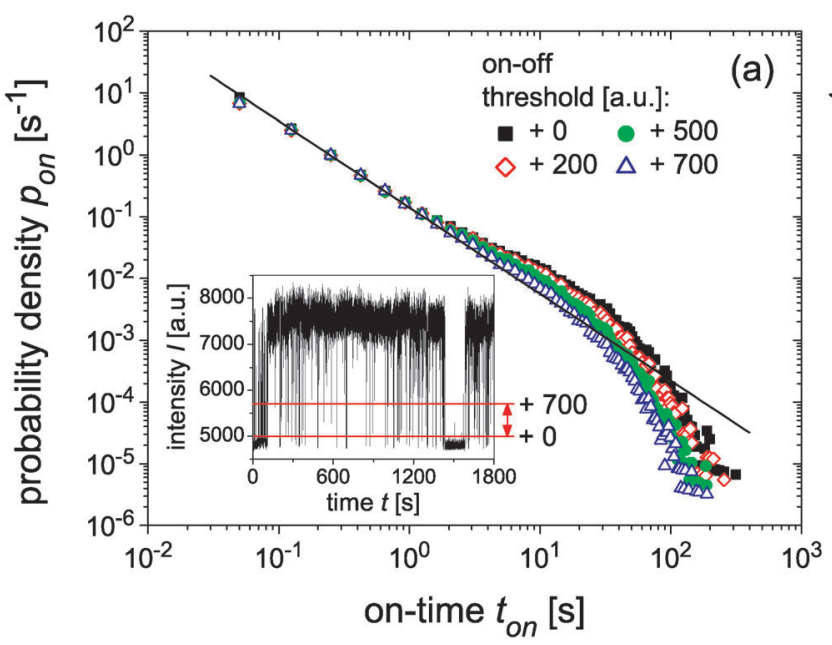

Both summands $p_{A}$ and $p_{B}$ relate to an inverse timescale allowing for dimensionless weighting factors $A$ and $B$. In Fig. 1(b) the fitting parameters are $\alpha_{\text {on }}=1.41 \pm 0.01$, which is again in agreement with on-time exponents found by other groups for this system, ${ }^{21,24,44} \Gamma_{\text {on }}=(9 \pm 1) 10^{-3} \mathrm{~s}^{-1}$ and $\gamma_{\text {on }}=(67 \pm 5) 10^{-3} \mathrm{~s}^{-1}$. The ratio of the weighting factors is given by $B / A=0.12 \pm 0.01$, meaning that the additional exponential function and the process responsible for this behaviour is about one order of magnitude less probable compared with the mechanism generating the power law dynamics. The reason for this deviation and its treatment by an exponential function will be discussed later.

At this point we emphasize again the wide range in blinking dynamics. As an essential condition, the large range in timescale of about four orders of magnitude enables us to observe small, but systematic deviations from a power law. In this context, another significant requirement is the determination of the intensity threshold separating the on- and off-events. In order to detect the on-time "hump" the threshold should be set as low as possible but still identifying the background as off-state. Fig. 2(a) clearly reveals the dependence of detectability of the on-time deviation on different thresholds. The on-time "hump" drops for increasing on/off discrimination levels and nearly vanishes in the case of high thresholds ( +700 a.u.). To visualize the extent of the examined thresholds the insets in Fig. 2 demonstrate the corresponding range within a typical time trace and its corresponding intensity histogram for a single QD. It is obvious that only a small shift of the threshold, compared to the absolute difference in intensity between the peaks for off- and on-events in the intensity histogram, makes the "hump" disappear. To examine the reason for this behaviour we plotted the same on-time distributions in Fig. 2(b) but without normalizing the probability densities in comparison to the normalized/scaled ones in Fig. 2(a). The absolute values for the probability density close to on-times of about $t_{\mathrm{on}} \approx 20 \mathrm{~s}$, where the on-time deviation reaches its maximum (see Fig. 2(a)), are unchanged for all distributions. This clearly reveals that the on-time deviation did not disappear, but is overlayed by an

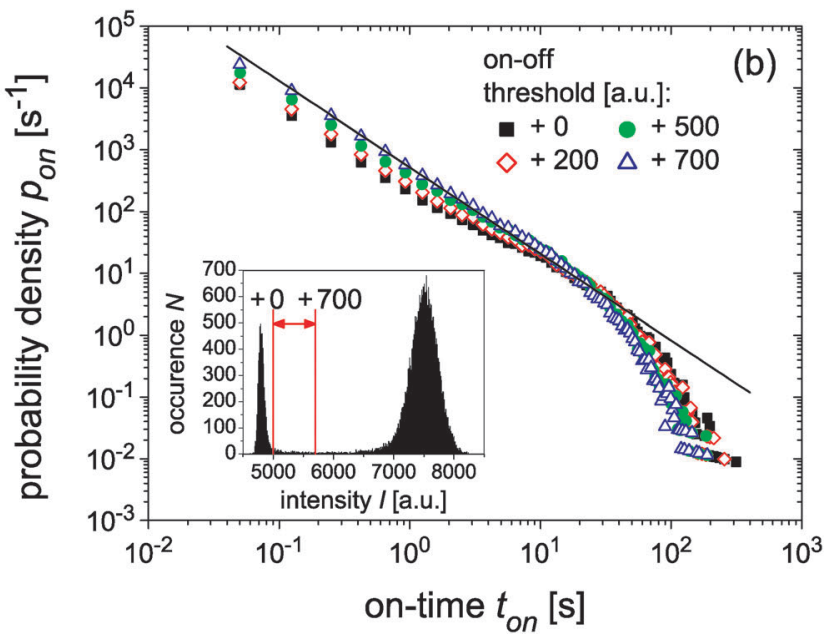

Fig. 2 On-time distributions of $100 \mathrm{CdSe} / \mathrm{ZnS}$ QDs on highly hydroxylated silicon oxide, already displayed in Fig. 1, as a function of different thresholds separating on- and off-events. The graphs compare the (a) normalized/scaled and (b) absolute probability densities of the on-events, respectively. The black lines denote an untruncated power law $p_{\text {on }}\left(t_{\text {on }}\right) \propto t_{\text {on }}^{-\alpha_{\text {on }}}$ with $\alpha_{\mathrm{on}}=1.4$. The insets illustrate the range of thresholds with respect to a typical time trace and its related intensity histogram for a single QD. 
increased probability density for shorter on-events $\left(t_{\text {on }}<20 \mathrm{~s}\right)$. These additional, but artificial short on-times arise through high thresholds which split longer on-events, consisting partly of low intensity values, into shorter ones and furthermore also reduce the amount of long on-events in Fig. 2(b). We have also analyzed the influence of a threshold lowering in order to observe an even stronger on-time "hump" (data not shown). However, this only results in highly overestimated probability densities for the shortest on-time durations already for small threshold reductions (up to minus 100 a.u.) due to a physically meaningless on-/off- event separation of background intensities. These findings indicate our thresholds to be well chosen and imply that the observed "hump" is not an artefact of data evaluation.

To get closer insights of the physical origin of the on-time deviation and to identify a possible dependence on the environment of the QDs, we analyzed the blinking dynamics on annealed silicon oxide surfaces with a reduced grade of hydroxylation and silanol group density, respectively. Fig. 3 compares both on- and off-time distributions of $\mathrm{CdSe} / \mathrm{ZnS}$ QDs on highly hydroxylated silicon oxide with those of annealed samples. In regard to the black line in the on-time statistics in Fig. 3(a) it is obvious that the deviation decreases in the case of annealed oxide surfaces, while the power law exponent $\alpha_{\text {on }}$ is (nearly) not affected (see inset of Fig. 3(a)). As can be seen from Fig. 3(a) the decrease of the "hump" is small for the moderate treatment at $500{ }^{\circ} \mathrm{C}$ (30 min) but more clearly visible in the case of long annealing at $800{ }^{\circ} \mathrm{C}(22 \mathrm{~h})$ or short annealing at $1000{ }^{\circ} \mathrm{C}(10 \mathrm{~min})$. Table 1 summarizes the fit parameters according to eqn (4). Obviously, the two power law parameters $A$ and $\alpha_{\text {on }}$ remain constant for all surface conditions which implies that the power law behaviour for short on-times is not affected by the silicon oxide treatment. Otherwise, both the probability amplitude $B$ and the rate $\gamma_{\text {on }}$ decrease and increase, respectively, in the case of reducing silanol densities. The observed increase of the truncation rate $\Gamma_{\text {on }}$ is possibly due to the difficulty in fitting $\Gamma_{\text {on }}$ and $\gamma_{\text {on }}$ independently. It is interesting to note that both high-temperature silicon oxide treatments, namely $800{ }^{\circ} \mathrm{C}(22 \mathrm{~h})$ and $1000{ }^{\circ} \mathrm{C}(10 \mathrm{~min})$, have nearly the same effect on the blinking dynamics indicating similar densities of silanols.

According to the above observations we suggest, that the on-time "hump" is related to the density of silanol groups on the silicon oxide surface. To prove this hypothesis we analyzed the PL intermittency of QDs on silicon oxide, which was initially annealed for $22 \mathrm{~h}$ at $800{ }^{\circ} \mathrm{C}$ but rehydroxylated via "Piranha" treatment in an ultrasonic bath. The related distribution is also displayed in Fig. 3(a) (red, unfilled triangles). In this case the on-time "hump" recovers and almost reaches its original magnitude before annealing (black squares, see also Table 1). From the fact that the silanol group density recovers nearly completely after rehydroxylation of the oxide ${ }^{36,38}$ this suggests a strong correlation between the density of silanol groups and the magnitude of the on-time "hump". Opposite to the on-times, the off-time distributions, plotted in Fig. 3(b), nearly show no dependence on the treatment of the silicon oxide or the silanol group density and could be fitted with a truncated power law according to eqn (3), which again results in $\alpha_{\text {off }}=1.71 \pm 0.03$ and $\Gamma_{\text {off }}=(21 \pm 3) 10^{-3} \mathrm{~s}^{-1}$.

To get further information about the physical background of the on-time "hump" and its relation to silanol groups we analyzed the time traces of all examined QDs with respect to their on-activity. For this purpose we calculated the on-time fraction $\Phi_{\text {on }}$ of each QD which we defined as the sum of all single on-time durations $t_{\mathrm{on}, i}$ within a certain time trace, normalized to its total length, i.e. the sum of all single on- and off-time durations $t_{\mathrm{off}, i}$

$$
\Phi_{\mathrm{on}}=\frac{\sum_{i} t_{\mathrm{on}, i}}{\sum_{i} t_{\mathrm{on}, i}+\sum_{i} t_{\mathrm{off}, i}}
$$

The on-time fractions of all evaluated QDs for high and low silanol densities are shown in Fig. 4(a) and (b), respectively. ${ }^{45}$ On both surfaces QDs show asymmetrically distributed ontime fractions with a maximum at high values (high on- but

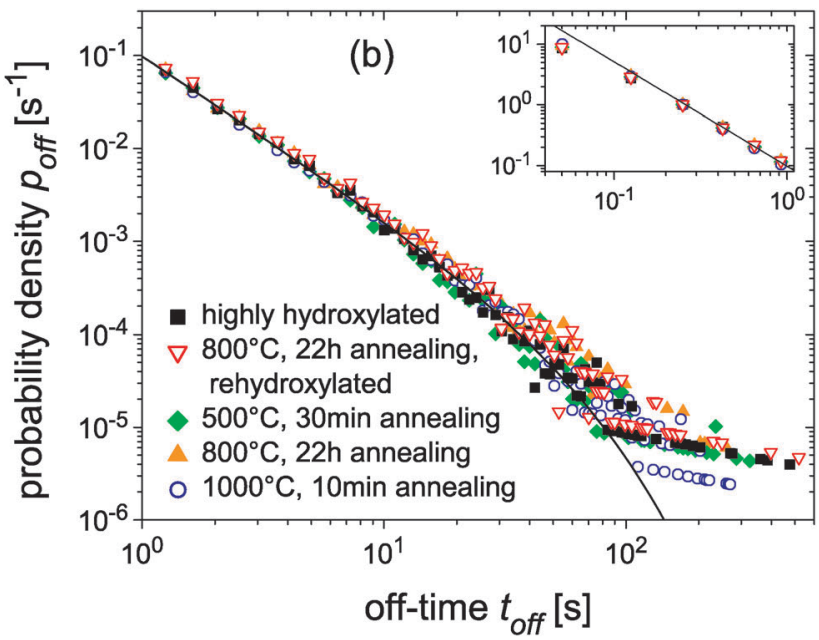

Fig. 3 Distributions for (a) on- and (b) off-times of at least $100 \mathrm{CdSe} / \mathrm{ZnS}$ QDs on silicon oxide surfaces with varying grades of hydroxylation and silanol group densities resulting from different preparation conditions. The insets display the time scale at very short on- and off-times. The black line in (a) corresponds to an untruncated power law $p_{\mathrm{on}}\left(t_{\mathrm{on}}\right) \propto t_{\mathrm{on}}^{-\alpha_{\mathrm{on}}}$ with $\alpha_{\mathrm{on}}=1.4$, whereas the fit of the off-times in (b) follows eqn (3) with $\alpha_{\text {off }}=1.71$ and $\Gamma_{\text {off }}=21 \times 10^{-3} \mathrm{~s}^{-1}$. 
Table 1 Fitting parameters ${ }^{a}$ of on-time distributions of QDs on silicon oxide surfaces with different pre-treatment

\begin{tabular}{lllclcr}
\hline Silicon oxide treatment & $A\left[10^{-3}\right]$ & $\alpha_{\text {on }}$ & $\Gamma_{\text {on }}\left[10^{-3} \mathrm{~s}^{-1}\right]$ & $B\left[10^{-3}\right]$ & $B / A$ & $\gamma_{\text {on }}\left[10^{-3} \mathrm{~s}^{-1}\right]$ \\
\hline Highly hydroxylated & $143 \pm 1$ & $1.41 \pm 0.01$ & $9 \pm 1$ & $17.5 \pm 1.2$ & $0.12 \pm 0.01$ & $67 \pm 5$ \\
$800^{\circ} \mathrm{C}, 22 \mathrm{~h}$ annealing and rehydroxylation & $138 \pm 3$ & $1.44 \pm 0.02$ & $11 \pm 1$ & $14.2 \pm 1.0$ & $0.10 \pm 0.01$ & $68 \pm 6$ \\
$500^{\circ} \mathrm{C}, 30$ min annealing & $136 \pm 4$ & $1.43 \pm 0.03$ & $18 \pm 2$ & $13.5 \pm 0.8$ & $0.10 \pm 0.01$ & $84 \pm 7$ \\
$800{ }^{\circ} \mathrm{C}, 22 \mathrm{~h}$ annealing & $136 \pm 4$ & $1.44 \pm 0.02$ & $22 \pm 3$ & $9.4 \pm 1.1$ & $0.07 \pm 0.01$ & $123 \pm 13$ \\
$1000^{\circ} \mathrm{C}, 10$ min annealing & $141 \pm 3$ & $1.42 \pm 0.02$ & $24 \pm 3$ & $9.9 \pm 1.3$ & $0.07 \pm 0.01$ & $133 \pm 16$ \\
${ }^{a}$ According to eqn (4). & & & & & \\
\hline
\end{tabular}

low off-fractions, see also inset of Fig. 2(a)). In comparison, QDs on highly hydroxylated silicon oxide show larger on-time fractions than those on strongly annealed oxide which is also apparent from their corresponding average values $\overline{\Phi_{\text {on }}}$ of 86.0 and $69.4 \%$, respectively. However, the distribution peaks sharply at about $\Phi_{\text {on }} \approx 0.95$ for the hydroxylated silicon oxide whereas it is broad with a maximum at $\Phi_{\text {on }} \approx 0.8$ in the case of a surface with a low silanol group density.

Additionally, we have sorted the 100 evaluated QDs in both cases according to increasing on-time fractions prior to a
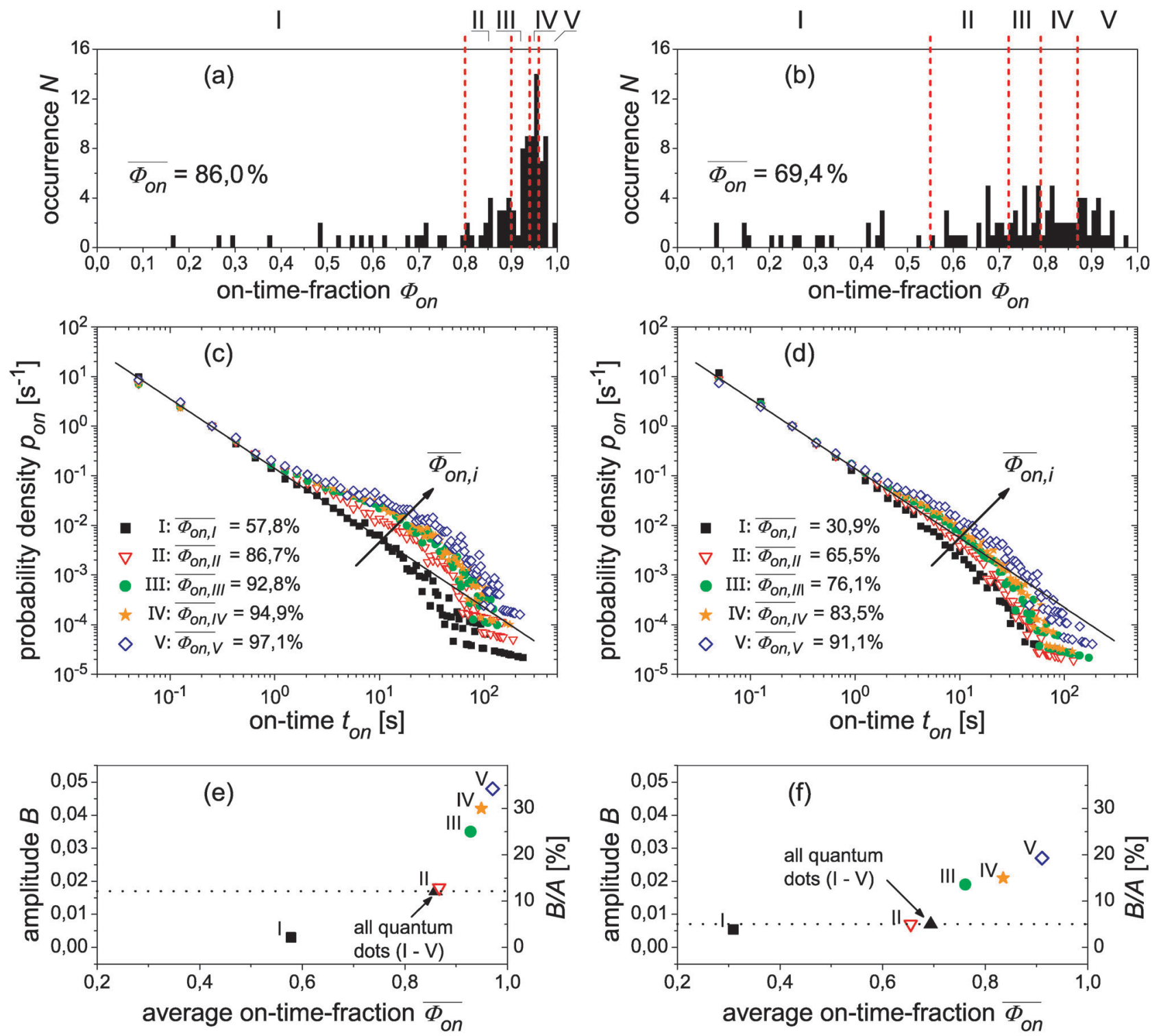

Fig. 4 Classification of $\mathrm{CdSe} / \mathrm{ZnS}$ QDs according to increasing on-time fractions $\Phi_{\text {on }}$ on highly hydroxylated (left) and strongly annealed $\left(1000{ }^{\circ} \mathrm{C}\right.$, right) silicon oxide. Sections (a) and (b) display the distributions and average values of on-time fractions. For both systems all $100 \mathrm{QDs}$ have been divided into five commensurate groups "I"-"V" (separation denoted by dashed lines). Related on-time distributions are shown in sections (c) and (d). The black line denotes the untruncated power law with an exponent $\alpha_{\text {on }}=1.4$. Sections (e) and (f) plot the amplitude $B$ (and the amplitude ratio $B / A$, see eqn (4)) as a function of the average on-time fraction $\overline{\Phi_{\text {on }}}$ highlighting the magnitude of the "hump" before (dotted line, filled triangle) and after grouping of the QDs. 
classification in commensurate groups "I"-"V", each consisting of 20 particles (separation visualized by vertical dashed lines in Fig. 4(a) and (b)). The on-time distributions and the average on-time fractions for these groups are given in Fig. 4(c) and (d). The increase of the on-time "hump" with increasing average on-time fraction (increasing group number) is evident and supports a correlation between the magnitudes of "hump" and on-time fraction. For a more quantitative access we have determined the amplitude $B$ of the exponential function in eqn (4) for all groups, illustrated in Fig. 4(e) and (f). Groups "II" nearly reproduce the on-time distributions gained for all (ungrouped) QDs (Fig. 3(a)) which is indicated by the horizontal dotted lines and black (filled) triangles in Fig. 4(e) and (f). In contrast, groups "I" with the lowest average on-time fractions do not show any "hump", whereas an enhancement becomes clearly visible for groups "III"-"IV". This means that the extent of the "hump" varies for different QDs but its manifestation can be correlated both with the silanol group density and the on-time fraction.

Finally, we examined the relationship between the amplitude of the "hump" and the PL intensities of the corresponding on-time events. To proceed, we have correlated the duration $t_{\mathrm{on}}$ of each on-event of an individual time trace to its averaged intensity $\overline{I_{\mathrm{on}}}$. In order to make a comparison between different QDs (with different PL intensities) feasible, we related these intensities to the maxima of "background" $\left(\overline{I_{\mathrm{on}}}=0\right)$ and "on"-peak $\left(\overline{I_{\text {on }}}=1\right)$ in the intensity histogram ( $c f$. inset of Fig. 2(b)) for each time trace. Fig. 5 shows typical graphs for a single QD on highly hydroxylated (Fig. 5(a)) and highly annealed (Fig. 5(b)) silicon oxide. Although the data points for the highly annealed system show a broad distribution, both plots in Fig. 5(a) and (b) exhibit increasing PL intensities with increasing on-times. Moreover, on-events which are related to the "hump" ( $\left.1 \mathrm{~s}<t_{\text {on }}<100 \mathrm{~s}\right)$ are characterized by average PL intensities close to the maximum of the on-peak $\left(\overline{I_{\mathrm{on}}} \approx 1\right)$. Therefore, larger "humps" result in an increased occurrence of high PL intensities which is obvious by a comparison of the intensity distributions given in the insets in Fig. 5(a) and (b) (see also accumulation of data points with high intensities in Fig. 5(a)). To generalize this trend we have plotted the average intensity distributions for all QDs in Fig. 5(c) and (d). From there a larger fraction of high intensities is clearly visible for the system with the larger "hump".

Let us summarize the experimental results. Correlated with the density of silanols we observe both a broad distribution of on-times as expressed by a power law and an additional exponential component (eqn (4)) with a typical time scale of $\gamma_{\mathrm{on}}{ }^{-1}=(7-15) \mathrm{s}$. This component comes along with high PL intensities. Nevertheless, not all of the single QDs in a given sample are affected by silanol groups (see Fig. 4 and 5(b)). This is reasonable, since it is known that silanols are not homogeneously distributed on the silicon oxide surface. ${ }^{47,48}$ The power law coefficients $\alpha$ (both for on- and off-times) are not influenced by the silanol density, whereas the cut-off time $\Gamma_{\text {on }}{ }^{-1}$, the amplitude $B$ of the "hump" and its decay time $\gamma_{\text {on }}{ }^{-1}$ decrease when reducing the silanol density. Concluding this part, CdSe/ZnS QDs can tentatively be divided in two classes. One class is not influenced by silanols (a typical representative is shown in Fig. 5(b)) and is characterized by a power law with a relatively short truncation time. Depending on the local silanol density the second class is described by a superimposed exponential term (on-time "hump") and a prolongation of the truncation time. Therefore, within optical resolution, QDs can be used as probes for the local silanol density.

To explain why the on-time "hump" appears and why it is related to the silanol density, we refer to the trapping model introduced by Verberk et al. ${ }^{35}$ which was worked out in more detail by Chon et al. ${ }^{20}$ In this model the dynamics of PL intermittency is explained in terms of light induced ionisation of QDs by tunnelling processes of electrons from the core of the nanocrystals to trap states outside (including the interfaces). In the simplest case of an uncharged QD excitons recombine radiatively ("on-state" with maximal PL intensity). If the particle becomes charged by tunnelling of the electron to trap states in the environment, leaving a delocalized hole within the QD (charged core), radiationless recombination pathways deactivate subsequently generated excitons ${ }^{49-51}$ leading to a quenching of the PL ("off-state"). Therefore, such an off-state persists (off-time) until the electron tunnels back. The probability of this tunnelling process $P_{r}\left(r, V_{t}\right)$ of a particle (with the respective effective mass $m_{\text {eff }}$ ) between two discrete states is given by the distance $r$ between these states and the magnitude of the corresponding energy barrier $V_{t}$ according to

$$
P_{r}\left(r, V_{t}\right) \propto \exp \left(-\frac{2 r}{\hbar} \sqrt{2 m_{\mathrm{eff}} V_{t}}\right)
$$

Since there is a broad distribution of "off-states" the related off-times follow power law dynamics ${ }^{16}$ whereby the truncation for long times simply results from a finite density of trap states. Despite this broad distribution of trap states, we have to expect an exponential distribution of on-times (tunnelling from one single initial state). From the discrepancy between this expectation and the experimentally observed power law distribution of "on-states" a second mechanism of switching (tunnelling) has to be thought of. On the one hand, this might be related to fluctuations of accessible trap states causing different tunnelling rates for the electron. Another possibility is related to the localisation of the remaining hole (after electron tunnelling) in trap states within the QD (most likely at the interface or in the $\mathrm{ZnS}$ shell). In that case the PL intensity will be partly restored depending on the charging conditions and gives rise to PL intensities intermediate between the bright, uncharged on-state and the completely quenched off-state (delocalized hole in the CdSe core). Hence, the time before the hole is delocalized again determines the duration of an on-event (on-time) with intermediate PL intensity. Since this process is related to tunnelling from a distribution of hole-trap states to the core the on-times also become power law distributed. The overall model for an interrelated tunnelling of hole and electron is shown in Fig. 6. Taking hole traps into account the on-time power law dependence and the broad range of intermediate intensities can be explained. ${ }^{14,15}$

Recently it has been shown that the off-time power law exponent $\alpha_{\text {off }}$ depends on the dielectric properties of the environment. ${ }^{23}$ As can be concluded from Fig. 3(b) the silanol 

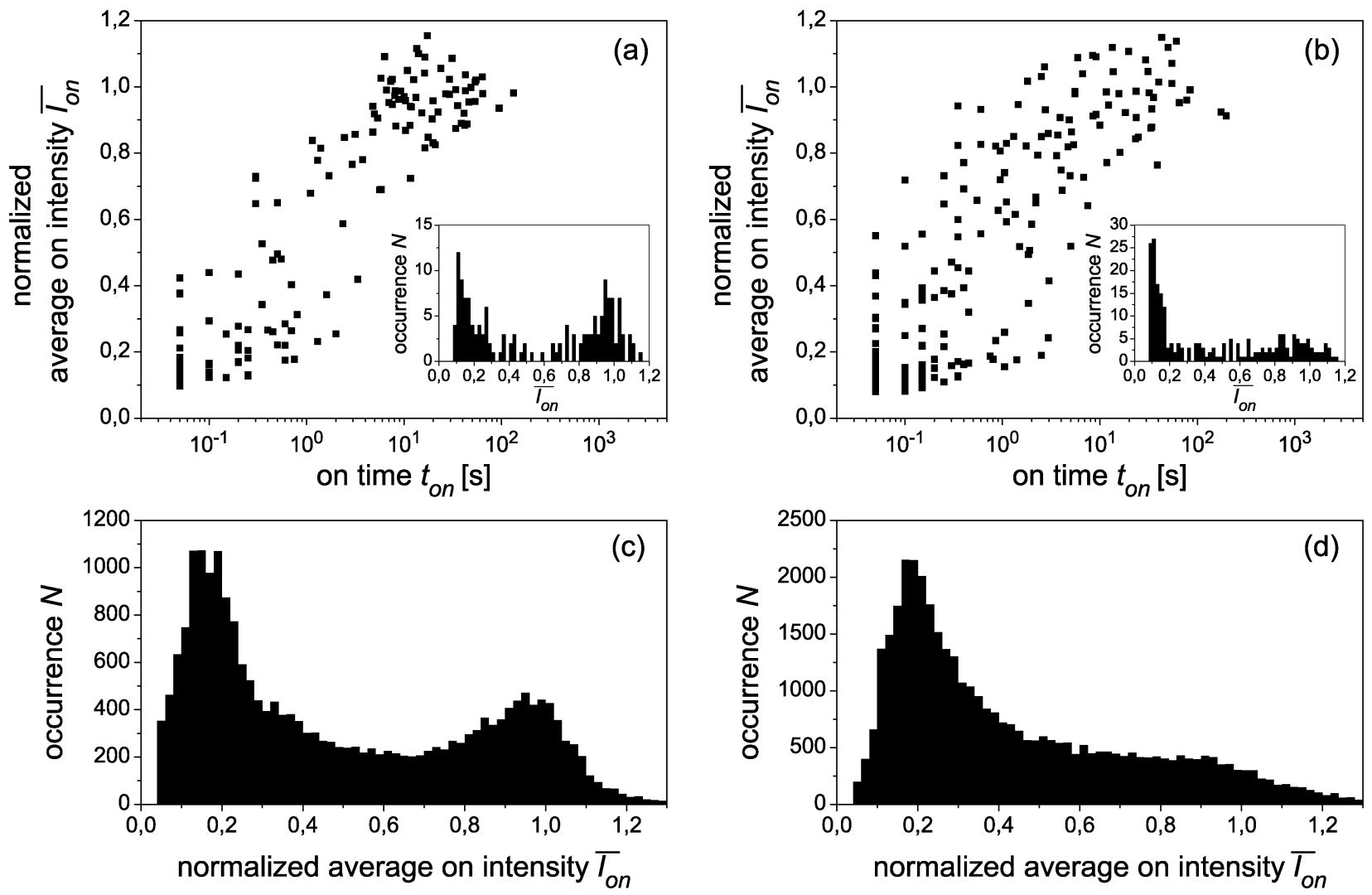

Fig. 5 Correlation between average PL intensity $\overline{I_{\mathrm{on}}}$ and length $t_{\mathrm{on}}$ for each on-event of a typical single QD on a highly hydroxylated (a) and highly annealed (b) silicon oxide surface, respectively. All intensities are normalized with respect to the maximum of the "background"- $\left(\overline{I_{\mathrm{on}}}=0\right)$ and the "on"-peak $\left(\overline{I_{\mathrm{on}}}=1\right)$ in the intensity histogram ( $c f$. inset of Fig. 2(b)) for each time trace. The insets in (a) and (b) show the corresponding intensity distributions. Sections (c) and (d) depict the distributions for all evaluated QDs on highly hydroxylated and highly annealed silicon oxide, respectively.

density has a negligible effect on $\alpha_{\text {off }}$ and $\Gamma_{\text {off }}$. However, on-time distributions clearly depend on the silanol density. While $\alpha_{\text {on }}$ remains constant, we took into account a superimposed exponential contribution of trap sites. This contribution is related with obviously high PL levels. Comparing all data it can be clearly ruled out that eqn (4) simply relates to a superposition of two distinct classes of QDs (featuring either power law or exponential distributed on times) since, as shown in Fig. 5(a), also a single QD clearly exhibits both components. In the context of the trapping model a single exponential function added to the truncated power law is a strong evidence for the presence of a discrete and well defined trap state depicted schematically in Fig. 6. Following this conjecture the observed on-time "hump" (single exponential component) is related to an enhanced localisation probability of a hole in a well defined trap. The correlation between this "hump" and the average silanol density proves the significant role of the Si-bounded hydroxyl groups. The more silanol groups are present the stronger the "hump" emerges. Therefore, a silanol induced hole trap is most likely responsible for the described on-time "hump" formation.

How can a silanol group induce a well defined hole trap state which, simultaneously, results in efficient PL of the QD? To answer this question one has to keep in mind that within a given sample not all of the single QDs are related to such a well defined trap. This heterogeneity is easily explained by the fact

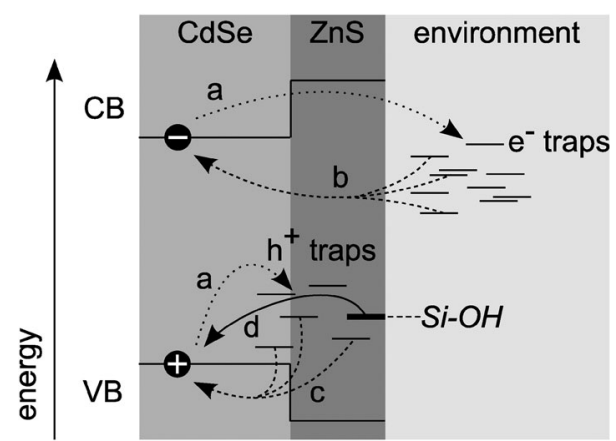

Fig. 6 Scheme of energy levels of a $\mathrm{CdSe} / \mathrm{ZnS}$ QD and trap states for electrons (environment) and holes (shell). The arrows denote trapping and detrapping processes whose dynamics are described by single exponential (a and d) and (truncated) power law (b for off-times, c for on-times) distributions, respectively. The process indicated by $\mathrm{d}$ relates to a well defined, silanol induced hole trap with enhanced localisation probability (bold line) inducing the on- time "hump".

that silanol groups do not homogeneously cover a silicon oxide surface. ${ }^{47,48}$ Creating a well defined trap needs a well defined interaction of a silanol group with a QD. Thus, one essential prerequisite is that a QD and specifically the $\mathrm{ZnS}$ shell has to be in close contact with at least one silanol group. This is, as shown in Fig. 7, only possible for steric reasons if the TOPO ligands of the QDs are at least partly removed. 


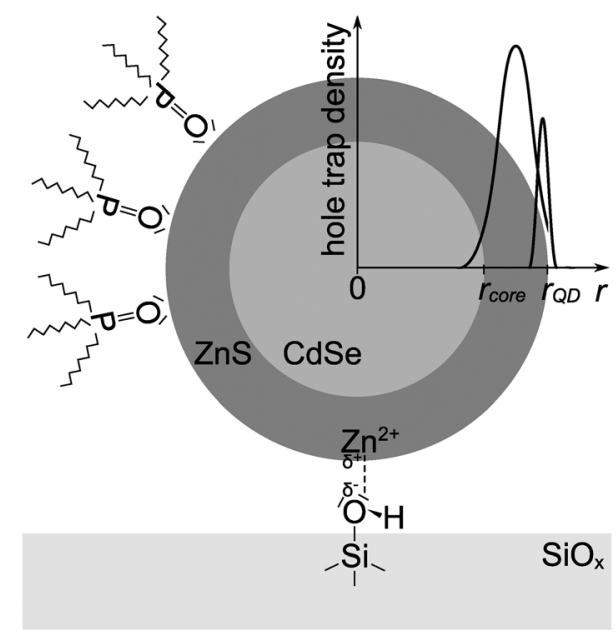

Fig. 7 Sketch of the (well defined) electrostatic bond between a silanol group and a partly charged $\mathrm{Zn}^{2+}$ atom at the $\mathrm{ZnS}$ surface of the QD (bottom). The radially distributed hole trap density (projected into the drawing plane) schematically shows the well defined and narrow distribution of hole trap states at the $\mathrm{ZnS}$ outer surface. Additionally, a broad distribution of hole trap states related to lattice disorder within the $\mathrm{ZnS}$ shell is depicted. Note that the corresponding widths of the two trap distributions are considered to have a large variance. Furthermore, the only partial coverage with TOPO ligands is indicated.

In that case $\mathrm{Si}-\mathrm{OH}$ can form an electrostatic bond with a $\mathrm{Zn}^{2+}$ atom at the outer surface of the $\mathrm{ZnS}$ shell in a similar manner as for (TOPO) ligands. ${ }^{52}$ It should be mentioned that a coordinated covalent bond between $\mathrm{Si}-\mathrm{OH}$ and $\mathrm{Zn}^{2+}$ is also possible. Such bonds subsequently facilitate the localisation of a hole at or close to the bond at the outer sphere of the $\mathrm{ZnS}$ shell. At the same time the overlap of the wave functions of the localized hole and subsequently excited excitons is reduced owing to the relative large distance resulting in a high PL intensity. According to eqn (6) this long distances (at least $\approx 1 \mathrm{~nm}$ shell thickness) will favour relatively long trapping times for the hole and thus long on-times will be observed (more often). With respect to chemical aspects, silanol groups act as hole acceptors. Instead of silanols it is also conceivable that negatively charged, deprotonated silanol groups (see reaction scheme (2)) deepen existing hole traps or generate new ones with increased localisation energies for holes.

Finally we like to remark that the clearly observed on-time truncation of the power law (which is hardly, if at all, evident for off-times) is probably related to the finite range for hole traps which is due to the finite thickness of the $\mathrm{ZnS}$ shell of about $1 \mathrm{~nm}$. This is in accordance with variations of the on-time distribution with different shells and ligands as previously reported. ${ }^{20}$

\section{Conclusions}

In summary, this work demonstrates the complexity of the blinking dynamics of single CdSe/ZnS QDs and the underlying physical mechanisms. For the first time it is disclosed that QDs on a silicon oxide surface with varying grades of hydroxylation and silanol group densities show on-time distributions which are characterized by the commonly observed (truncated) power law dependence but are also superimposed by a single exponential function. This deviation from the power law behaviour depends on the density of the silanol groups anchoring to a QD surface and is further correlated with large on-time fractions and high photoluminescence intensities. Therefore, an interaction between QDs and silanols was postulated which generates well defined new or modified hole traps with an enhanced localisation probability at the outer $\mathrm{ZnS}$ interface. This explanation is consistent with the trapping model originally proposed by Verberk et al. ${ }^{35}$ which takes both hole and electron tunnelling into account. The off-time distributions do not show modifications of the power law dependence with respect to differently prepared silicon oxide surfaces. This supports the assumption that silanols do not affect electrons tunnelling back to the QD. Further modelling will be necessary to investigate the interdependence of on- and off-statistics.

\section{Acknowledgements}

We acknowledge helpful discussions with R. Schmidt, TU Chemnitz.

\section{References}

1 W. E. Moerner and L. Kador, Phys. Rev. Lett., 1989, 62, 2535-2538.

2 M. Orrit and J. Bernard, Phys. Rev. Lett., 1990, 65, 2716-2719.

3 M. Nirmal, B. O. Dabbousi, M. G. Bawendi, J. J. Macklin, J. K. Trautman, T. D. Harris and L. E. Brus, Nature, 1996, 383 802-804.

4 M. Kuno, D. P. Fromm, H. F. Hamann, A. Gallagher and D. J. Nesbitt, J. Chem. Phys., 2000, 112, 3117-3120.

5 K. T. Shimizu, R. G. Neuhauser, C. A. Leatherdale, S. A. Empedocles, W. K. Woo and M. G. Bawendi, Phys. Rev. B: Condens. Matter, 2001, 63, 205316.

6 T. Basché, S. Kummer and C. Bräuchle, Nature, 1995, 373, $132-134$.

7 X. S. Xie and R. C. Dunn, Science, 1994, 265, 361-364.

8 J. Schuster, F. Cichos and C. von Borczyskowski, Appl. Phys. Lett., 2005, 87, 051915.

9 R. M. Dickson, A. B. Cubitt, R. Y. Tsien and W. E. Moerner, Nature, 1997, 388, 355-358.

10 C. Bradac, T. Gaebel, N. Naidoo, M. J. Sellars, J. Twamley, L. J. Brown, A. S. Barnard, T. Plakhotnik, A. V. Zvyagin and J. R. Rabeau, Nat. Nanotechnol., 2010, 5, 345-349.

11 D. A. V. Bout, W. T. Yip, D. Hu, D. K. Fu, T. M. Swager and P. F. Barbara, Science, 1997, 277, 1074-1077.

12 J. M. Lupton, Adv. Mater., 2010, 22, 1689-1721.

13 H. Lin, R. Camacho, Y. Tian, T. E. Kaiser, F. Würthner and I. G. Scheblykin, Nano Lett., 2010, 10, 620-626.

14 K. Zhang, H. Chang, A. Fu, A. P. Alivisatos and H. Yang, Nano Lett., 2006, 6, 843-847.

15 C. T. Yuan, W. C. Chou, D. S. Chuu, Y. N. Chen, C. A. Lin and W. H. Chang, Appl. Phys. Lett., 2008, 92, 183108.

16 F. Cichos, C. von Borczyskowski and M. Orrit, Curr. Opin. Colloid Interface Sci., 2007, 12, 272-284.

17 P. H. Sher, J. M. Smith, P. A. Dalgarno, R. J. Warburton, X. Chen, P. J. Dobson, S. M. Daniels, N. L. Pickett and P. O’Brien, Appl. Phys. Lett., 2008, 92, 101111.

18 X. Wang, X. Ren, K. Kahen, M. A. Hahn, M. Rajeswaran, S. Maccagnano-Zacher, J. Silcox, G. E. Cragg, A. L. Efros and T. D. Krauss, Nature, 2009, 459, 686-689.

19 Y. Chen, J. Vela, H. Htoon, J. L. Casson, D. J. Werder, D. A. Bussian, V. I. Klimov and J. A. Hollingsworth, J. Am. Chem. Soc., 2008, 130, 5026-5027.

20 B. Chon, S. J. Lim, W. Kim, J. Seo, H. Kang, T. Joo, J. Hwang and S. K. Shin, Phys. Chem. Chem. Phys., 2010, 12, 9312-9319.

21 D. E. Gómez, J. van Embden, J. Jasieniak, T. A. Smith and P. Mulvaney, Small, 2006, 2, 204-208. 
22 S. Hohng and T. Ha, J. Am. Chem. Soc., 2004, 126, 1324-1325.

23 A. Issac, C. von Borczyskowski and F. Cichos, Phys. Rev. B, 2005, 71, $161302(\mathrm{R})$.

24 A. Issac, PhD Thesis, Chemnitz University of Technology, 2006.

25 F. D. Stefani, W. Knoll, M. Kreiter, X. Zhong and M. Y. Han, Phys. Rev. B: Condens. Matter Mater. Phys., 2005, 72, 125304.

26 F. Koberling, A. Mews and T. Basché, Adv. Mater., 2001, 13, $672-676$.

27 A. K. Gooding, D. E. Gómez and P. Mulvaney, ACS Nano, 2008, 2, 669-676.

28 H. Huang, A. Dorn, G. P. Nair, V. Bulovic and M. G. Bawendi, Nano Lett., 2007, 7, 3781-3786.

29 S. J. Park, S. Link, W. L. Miller, A. Gesquiere and P. F. Barbara, Chem. Phys., 2007, 341, 169-174

30 M. Kuno, D. P. Fromm, S. T. Johnson, A. Gallagher and D. J. Nesbitt, Phys. Rev. B: Condens. Matter, 2003, 67, 125304.

31 J. Tang and R. A. Marcus, J. Chem. Phys., 2005, 123, 054704.

32 P. A. Frantsuzov and R. A. Marcus, Phys. Rev. B: Condens. Matter Mater. Phys., 2005, 72, 155321.

33 P. A. Frantsuzov, M. Kuno, B. Jankó and R. A. Marcus, Nat. Phys., 2008, 4, 519-522.

34 J. Zhao, G. Nair, B. R. Fisher and M. G. Bawendi, Phys. Rev. Lett., 2010, 104, 157403.

35 R. Verberk, A. M. van Oijen and M. Orrit, Phys. Rev. B: Condens. Matter, 2002, 66, 233202.

36 L. T. Zhuravlev, Colloids Surf., A, 2000, 173, 1-38.

37 W. Olthuis and P. Bergveld, IEEE Trans. Electr. Insul., 1992, 27, 691-697.

38 V. Dugas and Y. Chevalier, J. Colloid Interface Sci., 2003, 264, 354-361.

39 Y. Kim, N. W. Song, H. Yu, D. W. Moon, S. J. Lim, W. Kim, H. J. Yoona and S. K. Shin, Phys. Chem. Chem. Phys., 2009, 11, 3497-3502.
40 C. von Borczyskowski, F. Cichos, J. Martin, J. Schuster, A. Issac and J. Brabandt, Eur. Phys. J. Spec. Top., 2007, 144, 13-25.

41 K. L. Knappenberger, D. B. Wong, Y. E. Romanyuk and S. R. Leone, Nano Lett., 2007, 7, 3869-3874.

42 R. Verberk, J. W. M. Chon, M. Gu and M. Orrit, Phys. E., 2005, 26, 19-23.

43 R. Wang, Y. Zhang, C. Gan, J. Muhammad and M. Xia, Appl. Phys. Lett., 2010, 96, 151107.

44 C. H. Crouch, R. Mohr, T. Emmons, S. Wang and M. Drndic, J. Phys. Chem. C, 2009, 113, 12059-12066.

45 To compare on time fractions of different QDs it is necessary to ensure that both the observation times and delay times between the beginning of illumination of the dots and the beginning of recording their time traces are identical. This is due to statistical aging and nonergodicity of luminescent QDs leading to a decreasing probability for the occurrence of on events with increasing time of illumination ${ }^{46}$.

46 X. Brokmann, J. P. Hermier, G. Messin, P. Desbiolles, J. P. Bouchaud and M. Dahan, Phys. Rev. Lett., 2003, 90, 120601.

47 C. H. Lochmüller, A. S. Colborn, M. L. Hunnicutt and J. M. Harris, J. Am. Chem. Soc., 1984, 106, 4077-4082.

48 R. K. Iler, The Chemistry of Silica. Solubility, Polymerization, Colloid and Surface Properties, and Biochemistry, WileyInterscience, NewYork, 1st edn, 1979, ch. 6.

49 V. I. Klimov, A. A. Mikhailovsky, D. W. McBranch, C. A. Leatherdale and M. G. Bawendi, Science, 2000, 287, 1011-1013.

50 R. M. Kraus, P. G. Lagoudakis, J. Müller, A. L. Rogach, J. M. Lupton, J. Feldmann, D. V. Talapin and H. Weller, J. Phys. Chem. B, 2005, 109, 18214-18217.

51 L. W. Wang, M. Califano, A. Zunger and A. Franceschetti, Phys. Rev. Lett., 2003, 91, 056404.

52 P. Schapotschnikow, B. Hommersom and T. J. H. Vlugt, J. Phys. Chem. C, 2009, 113, 12690-12698. 